\title{
ANÁLISE WAVELETS DO COMPORTAMENTO TEMPORAL DAS TAXAS DE INTERNAÇÕES POR DIARREIA ANTES E APÓS A VACINA CONTRA O ROTAVIRUS NO ESTADO DO PARANÁ
}

\author{
Bianca Kodama $^{1}$, Eniuce Menezes de Souza ${ }^{2}$, Maria de Lourdes T. Masukawa ${ }^{3}$, Taqueco T. Uchimura ${ }^{3}$
}

\author{
Graduanda de Estatística, Dep. de Estatística, Pós-Grad. em Enfermagem, Dep. de Enfermagem \\ Universidade Estadual de Maringá \\ E-mails: biancakodama@hotmail.com, emsouza@uem.br, mltmasukawa@hotmail.com, \\ taqueco@gmail.com
}

\begin{abstract}
Resumo - Neste artigo, o comportamento espaço/temporal/sazonal das taxas de hospitalização por diarreia aguda em menores de um ano nas 6 macro regionais do estado do Paraná, no período de 2000 a 2010 é analisado a partir de metodologias tanto no domínio do tempo quanto frequência. Na análise de séries temporais, são frequentes os casos em que não é possível induzir a estacionariedade, de modo que a maioria dos métodos tradicionais para séries temporais não podem ser aplicados. Nesse sentido, a análise wavelets vem se mostrando como uma ferramenta importantíssima. Uma versão modificada das wavelets, chamada de não decimada, é invariante por translação e tem propiciado excelentes resultados na análise de séries temporais. É possível realizar uma decomposição da variância total da série temporal em diferentes escalas. Diferentemente das usuais wavelets decimadas, o mesmo número de coeficientes é mantido em cada escala na decomposição, permitindo que toda correspondência temporal seja mantida na análise. Uma vez que a variância de uma determinada série é decomposta em diferentes escalas, fenômenos anteriormente ocultos podem ser revelados, tais como sazonalidades, variações cíclicas, pontos de mudança, etc. Os resultados se mostraram promissores na investigação do comportamento das internações e do rotavirus, principalemente em relação à sazonalidade.
\end{abstract}

Palavras-chave—Wavelets não decimadas, Séries Temporais, Rotavirus, Sazonalidade.

\section{Introdução}

Mundialmente o rotavírus é considerado um dos mais importantes agentes causadores de gastroenterites e de óbitos em crianças menores de cinco anos. Estima-se que, a cada ano, ocorram 125 milhões de quadros diarreicos associados ao rotavírus, resultando em 418.000 a 520.000 óbitos, sendo $85 \%$ em regiões menos desenvolvidas.

Segundo o Ministério da Saúde ${ }^{2}$, no Brasil, a incidência de diarreias associadas ao rotavírus varia de $12 \%$ a $42 \%$ e estima-se que, cerca de 2.500 crianças menores de cinco anos morriam por ano até 2006. Em março de 2006, a vacina do rotavírus foi incluída no Programa Nacional de Imunização (PNI). Após 2006, a redução das internações por diarreia tem sido observada e seu impacto tem sido avaliado (LANZIERRI et al, 2010; CARMO et al., 2011, SOUZA et al., 2013). Entretanto, o comportamento espaço/temporal/sazonal das internações por diarreia ainda é bastante controverso e necessita de investigações (HASHIZUME, 2008; MELLI, 2009, PITZER et al., 2009).

Diante do exposto, o objetivo do presente estudo foi, utilizando os dados oficiais do DATASUS, analisar as taxas de hospitalização (THs) por diarreia aguda em menores de um ano no Estado do Paraná, no período de 2000 a 2010, apresentando como principal enfoque a análise do comportamento temporal das taxas de internação para as 6 macro regionais do estado. Devido a dependência/correlação serial dos dados ao longo do tempo, faz-se necessária uma análise adequada para séries temporais. Por outro lado, quando não é possível induzir a estacionariedade, a maioria dos métodos tradicionais não é suficiente ou não pode ser aplicada, além do fato de que muitos fenômenos não podem ser identificados diretamente nos dados no domínio do tempo. Nesse sentido, a análise wavelets vem se mostrando como uma ferramenta importantíssima, a qual permite a modelagem e/ou análise de tal série no domínio do tempo e frequencia simultaneamente para que periodicidades, sazonalidades, pontos de mudança e outras informações relevantes possam ser detectados.

Muitas séries temporais, embora não estacionárias, podem ser consideradas localmente estacionárias (Nason et al., 2000). Para esses processos, a estimação da função densidade espectral a partir do periodograma de wavelets permite uma análise detalhada da série e a investigação dos efeitos existentes na mesma.

\section{Objetivo}

Analisar o comportamento temporal das taxas de hospitalização por diarreia aguda em menores de um ano nas 6 macro regionais do estado do Paraná, no período de 2000 a 2010, a partir de metodologias tanto no domínio do tempo quanto frequência para que correlações, tendências, periodicidades, sazonalidades, pontos de mudança e outras informações relevantes possam ser detectados. 


\section{Wavelets}

As wavelets são ondas de curta duração com energia concentrada num intervalo de tempo (Graps, 1995) e por isso denominadas de funções de suporte compacto. São definidas no espaço de quadrado integrável sobre $\mathrm{R}, \mathrm{L}^{2}(\mathrm{R})$.

Um processo wavelet localmente estacionário, $\left\{X_{t, T}\right\}_{t=0, \ldots, T-1}, T=2^{M} \geq 1$, em que M é o nível de resolução mais alta, é um processo estocástico duplamente indexado com a representação no sentido de média quadrática dada por

$$
X_{t, T}=\sum_{j=0}^{M-1} \sum_{k=-\infty}^{\infty} w_{j, k, T} \psi_{j, k}(t) \varepsilon_{j, k},
$$

em que $\left\{\varepsilon_{j, k}\right\}$ é uma sequência de incrementos ortonormal aleatória, $\left\{\psi_{j, k}(t)\right\}_{j, k}$ é um conjunto de wavelets discretas não decimadas e $\left\{w_{j, k, T}\right\}$ é um conjunto de amplitudes.

A transformada wavelet discreta é dada por

$$
d_{j, k}^{\psi}=\sum_{t=0}^{T-1} X_{t} \psi_{j, k}\left(\frac{t}{T}\right),
$$

com $j=0,1, \ldots, M$ níveis de decomposição; $k=0,1, \ldots, 2^{j}$ e $T=2^{M}, M>0$. É denominada não decimada quando o número de amostras em todos os níveis de decomposição permanece o mesmo, não desprezando as informações redundantes e fazendo melhores estimativas do espectro.

Para as quantidades da representação (1), são especificadas três condições por Nason et al. (2000):

1. $E\left(\varepsilon_{j, k}\right)=0$;

2. $\operatorname{cov}\left(\varepsilon_{j, k}, \varepsilon_{l, m}\right)=\delta_{j, l} \delta_{k, m}$, em que $\delta_{j, l}$ é o delta de Kronecker;

3. A velocidade da evolução de $w_{j, k, T}$ é controlada, para que ele não desvie de uma função $W_{j}(z)$ para $z \in(0,1)$, assumindo

$$
\sup \left|\mathrm{w}_{\mathrm{j}, \mathrm{k}, \mathrm{T}}-\mathrm{W}_{\mathrm{j}}\left(\frac{\mathrm{k}}{\mathrm{T}}\right)\right| \leq \frac{C_{j}}{T},
$$

em que $\left\{C_{j}\right\}$ é um conjunto de constantes com $\sum_{j=0}^{M-1} C_{j}<\infty$ e $z=\frac{k}{T}$. Quanto menor a velocidade da evolução do processo, maior o conjunto de observações a serem agrupadas para obter boas estimativas do processo $W_{j}\left(\frac{k}{T}\right)=W_{j}(z)$.

Em analogia ao espectro de Fourier, $f(w)=|A(w)|^{2}$, em que $A(w)$ é a amplitude do processo na freqüência $w$, tem-se uma função similar, o espectro wavelet evolucionário ou função de densidade espectral.

A função de densidade espectral wavelet, a qual mede a energia local, ou seja, a contribuição à variância no nível de resolução $j$ e tempo $z$ (NASON, 1999), é definida como:

$$
S_{j}(z)=\left|W_{j}(z)\right|^{2}, z \in(0,1)
$$

Se a série temporal $\left\{X_{t}\right\}$ é um processo localmente estacionário, a autocovariância local wavelet $c_{T}(z, \tau)$ deve depender do tempo. Nason et al. (2000) mostra que essa autocovariância converge para $c(z, \tau)$ quando $T \rightarrow \infty$,

$$
\begin{gathered}
c_{T}(z, \tau)=\operatorname{cov}\left(X_{\lfloor z T\rfloor, T}, X_{\lfloor z T\rfloor+\tau, T}\right), \\
c(z, \tau)=\sum_{j=0}^{M-1} S_{j}(z) \psi_{j}(\tau)
\end{gathered}
$$

em que $\psi_{j}(\tau)$ é a função de autocorrelação wavelet em $(j, k)$,

$$
\psi_{j}(\tau)=\sum_{k} \psi_{j, k}(0) \psi_{j, k}(\tau),
$$

para todo $j>0$ e $\tau \in R$.

Para estimar a função de densidade espectral, um estimador é o periodograma wavelet,

$$
I_{k, T}^{j}=\left|d_{j, k ; T}\right|^{2}
$$

Desse modo, a partir do periodograma wavelet, tem-se uma decomposição de variâncias em multiescala da série temporal, ou seja, em diversos níveis de resolução $j$.

\section{Materiais e Método}

Os dados foram obtidos do Sistema de Informação Hospitalar do SUS (SIH-SUS) utilizando-se a décima revisão da Classificação Internacional de Doenças (CID-10), com os códigos A00 e A09.

Tais dados consistem de todas as crianças menores de 1 ano residentes nas seis macro regiões: Leste, Campos Gerais, Centro Sul, Oeste, Noroeste e Norte, no Estado do Paraná, que foram hospitalizadas pelo SUS por doenças diarréicas agudas no período de janeiro de 2000 a agosto de 2010, sendo que a partir de 2006 as crianças começaram a receber a vacina contra o Rotavirus.

O cálculo da taxa de hospitalização (TH) por diarreia aguda foi realizado dividindo-se o número de hospitalizações por diarreia aguda pela população de menores de 1 ano de idade e multiplicando-se o quociente por 10.000. Essa multiplicação é usual na área de saúde pelo fato dos valores das taxas de hospitalizações serem de pequena magnitude. Assim uma taxa de 10 casos, significa que ocorreram 10 internações para cada 10.000 crianças menores de 1 ano. Embora os dados da TH incluam diarreia aguda por diferentes causas, o rotavírus é o principal causador de tais problemas de gastroenterites ou diarreia. 
A análise desses dados foi feita a partir dos clássicos correlogramas, espectros de Fourier e wavelets utilizando o software estatístico R. Para detalhes sobre correlogramas e espectro de Fourier, pode-se consultar Chatifield (1999), dentre outros. Os princípios básicos de wavelts foram brevemente descritos na seção 3 .

Embora dados mais atuais estivessem disponíveis, foram dispensados nessa análise devido a necessidade de que o comprimento da série temporal fosse potência de $2\left(128=2^{7}\right)$ para a decomposição wavelets em multiescala. Embora fosse possível expandir as bordas, optou-se por não usar tais métodos para não influenciar as análises de periodocidades.

\section{Resultados}

Os resultados serão apresentados para cada uma das 6 macro regionais, as quais podem ser visualizadas na Figura 1.
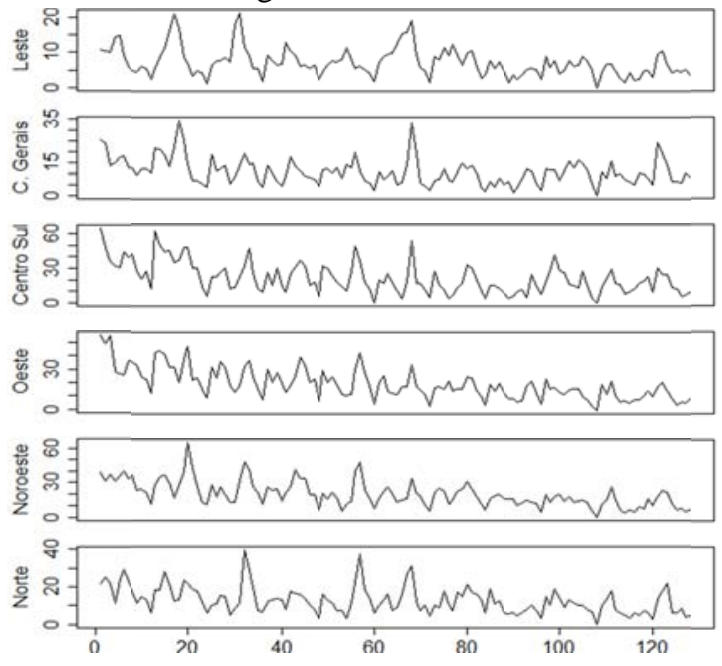

Figura 1: Série temporal das macrorregiões Leste, Campos Gerais, Centro Sul, Oeste, Noroeste e Norte.

Na Figura 2 são plotados o correlograma (a) e os periodogramas de Fourier para os períodos 2000-2010 (b), 2000-2005 (c), 2007-2010 (d) para a macrorregião Leste.

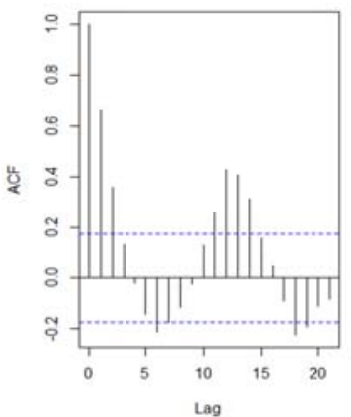

(a)

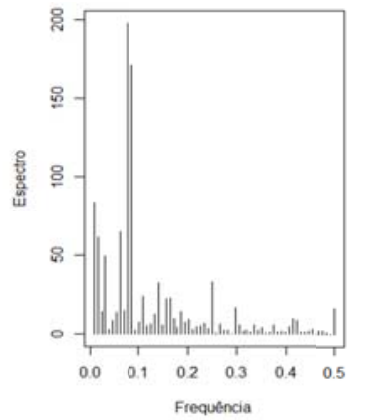

(b)

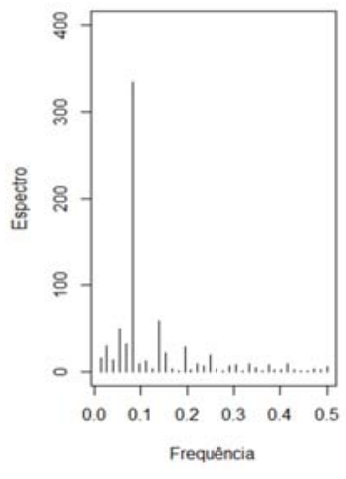

(c)

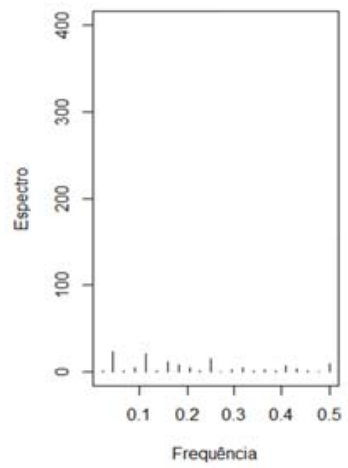

(d)
Figura 2: (a) Correlograma; (b) Periodogramas de Fourier 20002010 (c) Periodogramas de Fourier 2000-2005; (d) Periodogramas de Fourier 2007-2010 referentes às taxas de hospitalização por diarréia agudla da macrorregião Leste.

O gráfico da função de autocorrelação (correlograma) na Figura 2'a mostra que há uma sazonalidade de 12 meses para a macrorregião Leste. Essa sazonalidade é confirmada pelo periodograma de Fourier (Figura 2b), no qual as frequências que atingem os maiores valores do espectro são $f=0,078125$ e $f=0,0859373$. Como o período é o inverso da frequência, os dois picos referem-se às periodicidades de 12,8 e 11,6 anos, o que indica a presença de um comportamento sazonal anual. O periodograma da Figura $2 \mathrm{~b}$ foi feito para todo o período de dados. Devido ao fato da análise de Fourier indicar apenas a presença de sazonalidade, mas não indicar quando a mesma ocorre, é importante a visualização dos periodogramas antes (Figura 2c) e após (Figura 2d) a vacina. Nota-se que a sazonalidade é evidente apenas antes da vacina. Para uma análise mais detalhada em multiescala, foi plotado o espectro wavelets estimado pelo periodograma wavelet e o espectro global o qual representa a energia ou variância total em cada nível de resolução ou escala referente a este nível (Figura 3).

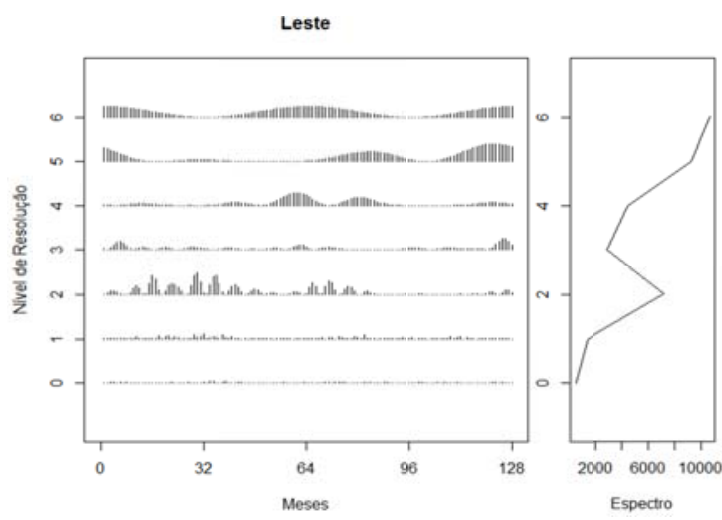

Figura 3: Periodograma wavelet es espectro global wavelet das taxas de hospitalização por diarréia aguda da macrorregião Leste. 
Observa-se na Figura 3 que a escala relativa ao nível de resolução 2 do Periodograma wavelet é a mais evidente. Esta escala é a mais próxima da periodicidade de 6 meses, apontando uma sazonalidade semestral. Embora na FAC e Periodograma de Fourier tenha sido indicada uma periodicidade anual, a análise de wavelets não está contraditória, uma vez que um comportamento semestral também se repete anualmente. Nota-se também, que a partir da vacina em 2006, 72 meses, tal comportamento sazonal da série diminuiu.

Para as macro regiões Campos Gerais, Centro Sul, Oeste, Noroeste e Norte, os correlogramas mostram que há uma sazonalidade de aproximadamente 6 meses. É confirmada pelos Periodogramas de Fourier, em que a frequência obtida foi de 0,1640625 e tempo de 6,095 meses para as macrorregiões mencionados.

Os gráficos dos periodogramas wavelets mostram que a escala relativa ao nível de resolução mais evidente é a 2. Assim como na macrorregião Leste, as outras macros também mostraram que o comportamento sazonal ficou menos evidente ou quase desapareceu após a vacina em 2006.

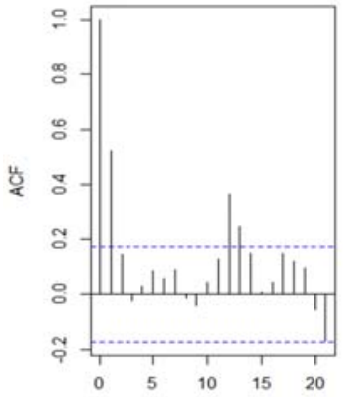

Lag

(a)

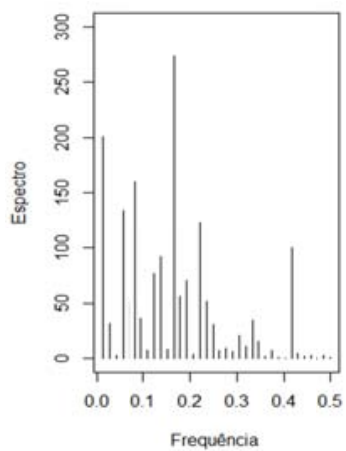

(c)

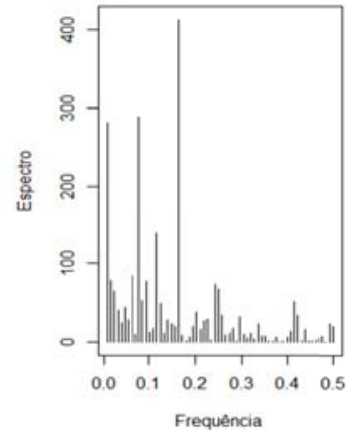

(b)

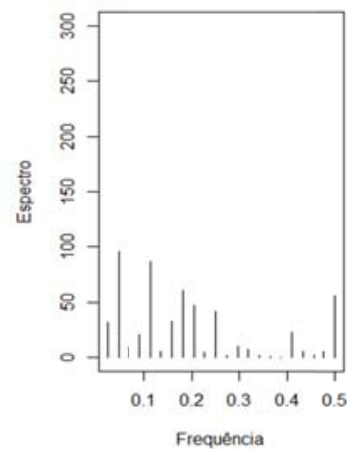

(d)
Figura 4: (a) Correlograma; (b) Periodogramas de Fourier 20002010 (c) Periodogramas de Fourier 2000-2005; (d) Periodogramas de Fourier 2007-2010 referentes às taxas de hospitalização por diarréia aguda da macrorregião Campos Gerais.

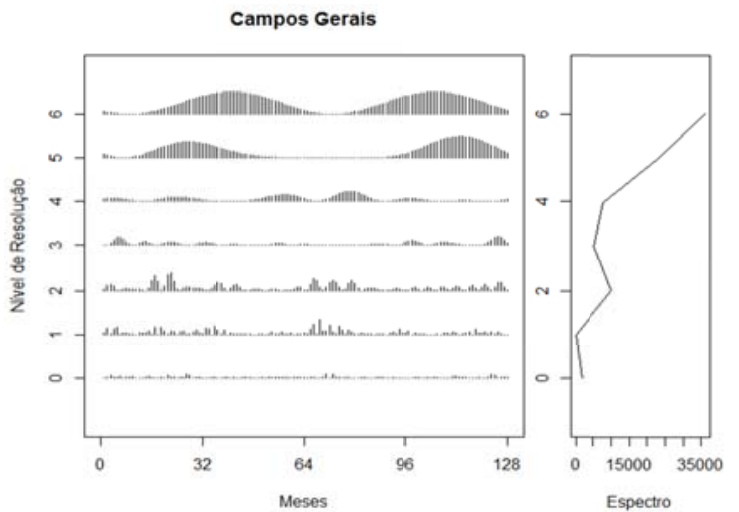

Figura 5: Periodograma wavelet das taxas de hospitalização por diarréia aguda da macrorregião Campos Gerais.

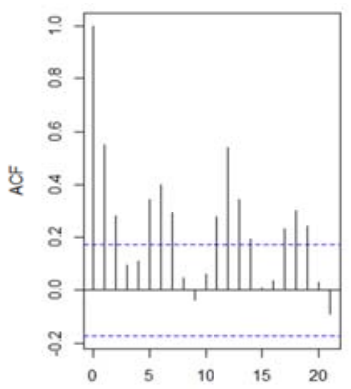

Lag

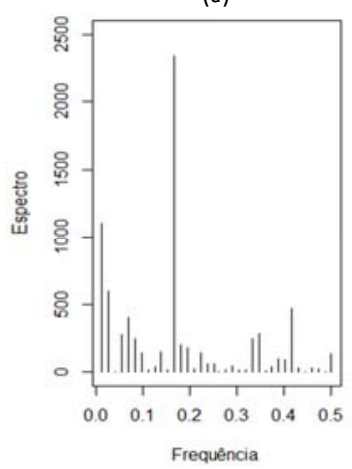

(c)

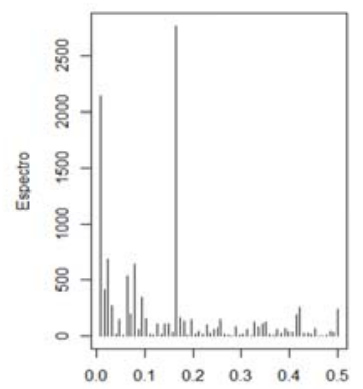

Frequencia

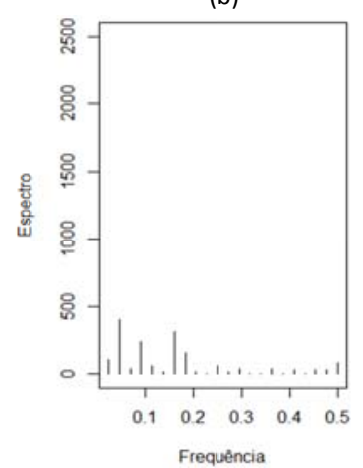

(d)
Figura 6: (a) Correlograma; (b) Periodogramas de Fourier 2000-

2010 (c) Periodogramas de Fourier 2000-2005; (d)

Periodogramas de Fourier 2007-2010 referentes às taxas de hospitalização por diarréia aguda da macrorregião Centro Sul.

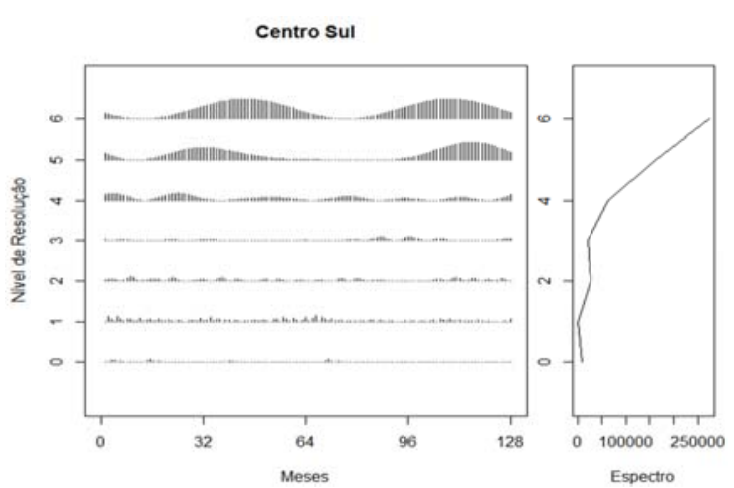

Figura 7: Periodograma wavelet das taxas de hospitalização por diarréia aguda da macrorregião Centro Sul. 


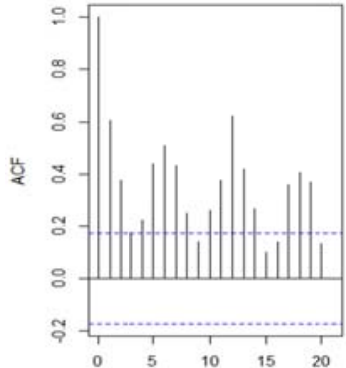

(a)

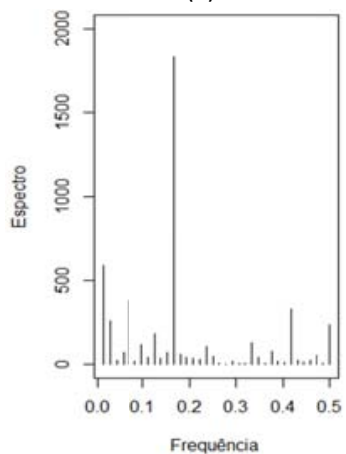

(c)

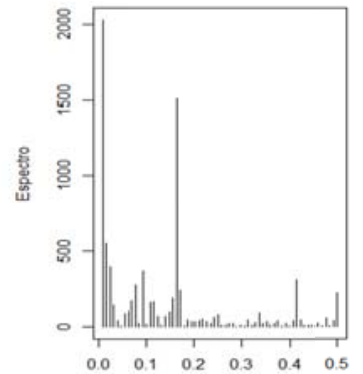

(b)

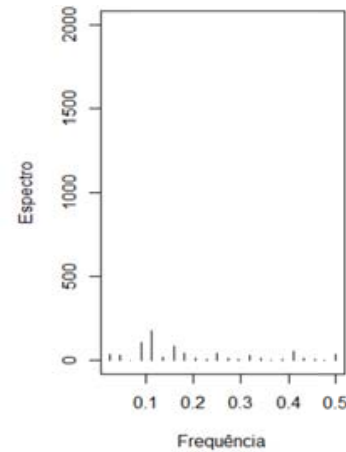

(d)
Figura 8: (a) Correlograma; (b) Periodogramas de Fourier 20002010 (c) Periodogramas de Fourier 2000-2005; (d)

Periodogramas de Fourier 2007-2010 referentes às taxas de hospitalização por diarréia aguda da macrorregião Oeste.

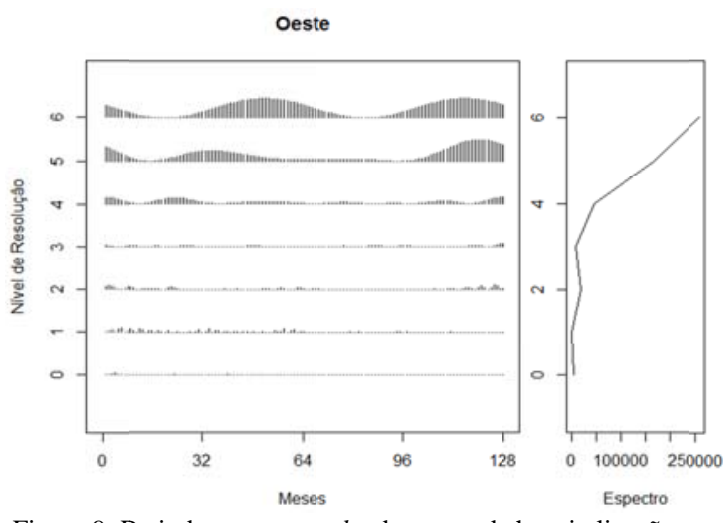

Figura 9: Periodograma wavelet das taxas de hospitalização por diarréia aguda da macrorregião Oeste

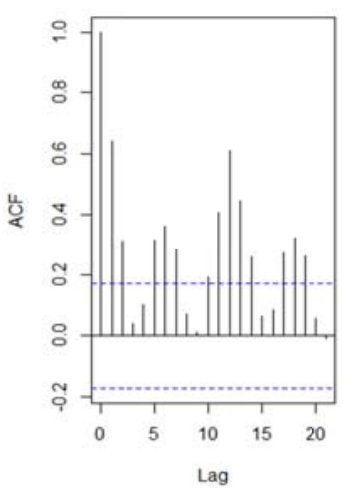

(a)

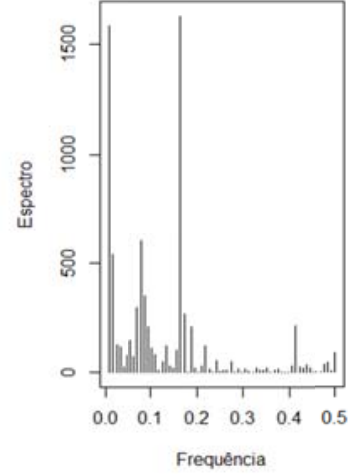

(b)

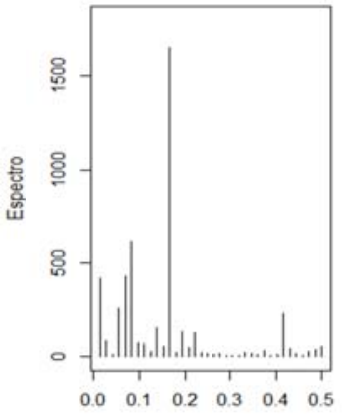

Frequência

(c)

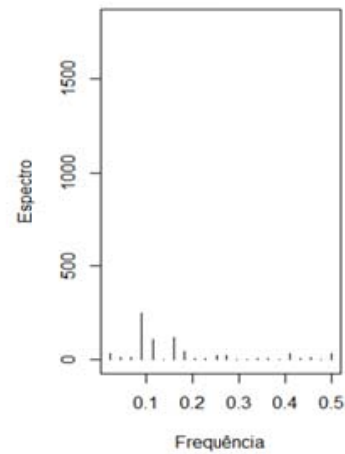

(d)
Figura 10: (a) Correlograma; (b) Periodogramas de Fourier 2000-2010 (c) Periodogramas dle Fourier 2000-2005; (d)

Periodogramas de Fourier 2007-2010 referentes às taxas de hospitalização por diarréia aguda da macrorregião Noroeste.

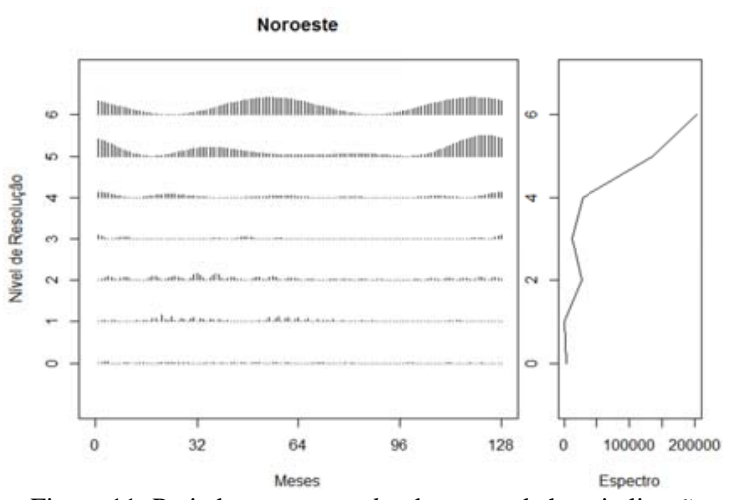

Figura 11: Periodograma wavelet das taxas de hospitalização por diarréia aguda da macrorregião Noroeste.

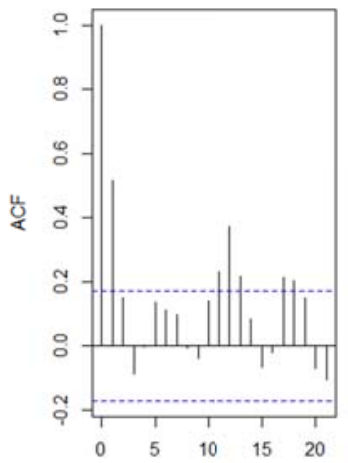

Lag

(a)

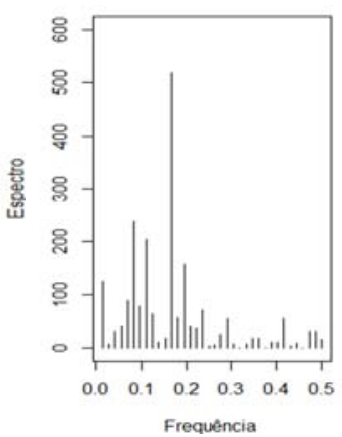

(c)

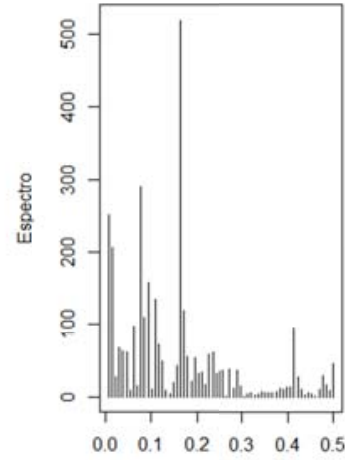

Frequência

(b)

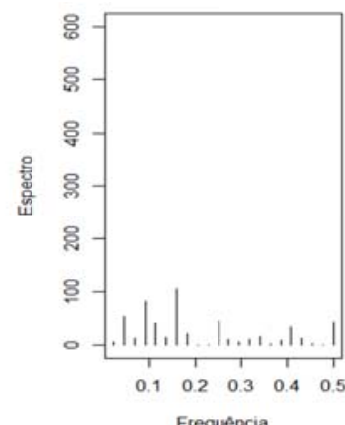

(d)
Figura 12: (a) Correlograma; (b) Periodogramas de Fourier 2000-2010 (c) Periodogramas de Fourier 2000-2005; (d) Periodogramas de Fourier 2007-2010 referentes às taxas de hospitalização por diarréia agudla da macrorregião Norte. 


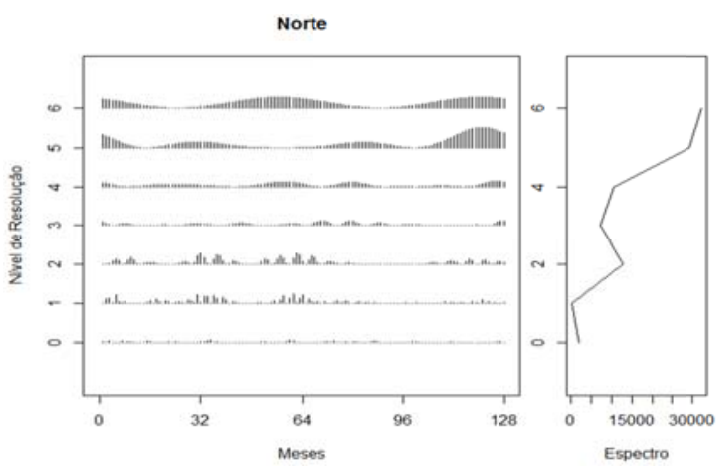

Figura 13: Periodograma wavelet das taxas de hospitalização por diarréia aguda da macrorregião Norte.

Observa-se em todas as macros regiões que as escalas relativas aos níveis de resolução 5 e 6 ficaram bem evidentes, mas o nível de resolução 6 representa os efeitos de 64 a 128 meses (5anos e 4 meses - 10 anos e 8 meses) enquanto o nível 5, os efeitos de 32 a 64 meses ( 2 anos e 8 meses - 5 anos e 4 meses). Tais níveis representam as variações suaves e de longo prazo da série, que para essa aplicação não capturam os efeitos de interesse, embora boa parte da energia da série esteja nesses dois níveis.

A Figura 14 mostra a escala referente ao nível de resolução 2 do periodograma wavelet, o qual foi mais evidente para todas as macrorregiões. Nota-se para todas as escalas a ocorrência de problemas de borda no final da série. No pacote wavethresh utilizado no software R para a TWND, a única opção para o tratamento de bordas é a periódica. Outras estratégias podem ser implementadas para evitar tal problema.

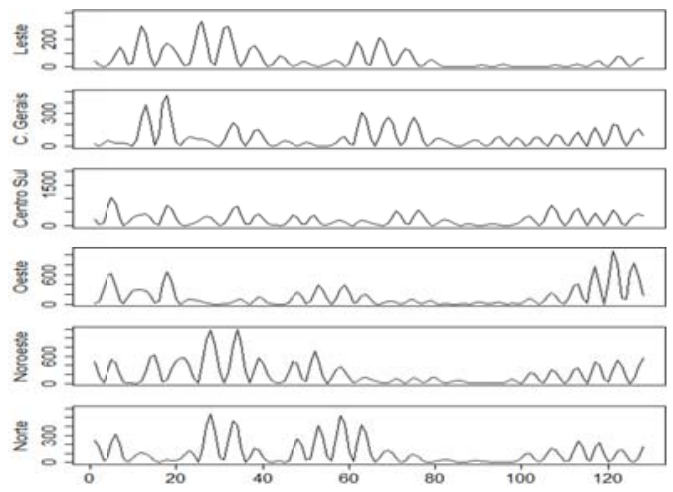

Figura 14: Escala relativa ao nível de resolução 2 do Periodograma wavelet das taxas de hospitalização por diarréia aguda por macrorregião.

\section{Conclusão}

Os resultados mostraram a presença de sazonalidade semestral e anual nas taxas de hospitalização, o que está de acordo com alguns trabalhos da literatura, os quais mostram que o rotavirus tem esse comportamento. Após a vacina, com as análises no domínio do tempo e frequência, verifica-se que esse comportamento sazonal fica bem menos explícito e praticamente desaparece para algumas macrorregionais.Esse fato reforça a eficácia da vacina em relação ao rotavírus, mesmo que este não esteja sendo avaliado diretamente.

\section{Agradecimentos}

A primeira autora agradece a bolsa de iniciação científica PIBIC/CNPq-Fundlação Araucária-UEM.

\section{Referências Bibliográficas}

CARMO, G. M. I., YEN, C., CORTES, J., SIQUEIRA, A. A., OIVEIRA, W. K, CORTES-ESCALENTE J. J., at al. Decline in Diarrhea Mortality and Admissions after Routine Childhood Rotavirus Immunization in Brasil: A Time-Series Analysis. PLoSMed.2011 Apr;8(4):e1001024. Epub 2’011. DOI: 10.1371 /journal.pmed.1001024

CHATFIELD, C. The Analysis of Times Series: An Introduction London: Chapman and Hall. 2003.

CURNS A. T, STEINER C. A., BARRET M, HUNTER K, WILSON E, PARASHAR U. D. Reduction in Acute Gastroenteritis Hospitalizations among US Children After Introduction of Rotavirus Vaccine: Analysis of Hospital. Discharge Datafrom 18 US States. J InfectDis, 201(11):1617-24, 2010.

HASHIZUME, M.; ARMSTRONG, B.; WAGATSUMA, Y.; FARUQUE, A. S. G.;

HAYASHI, T. e SACK, D. A. Rotavirus infections and climate variability in Dhaka, Bangladesh: a timeseries analysis. Epidemiol. Infect. (2008), 136, 12811289, 2007 Cambridge University Press.

MELLI, L. C. F. L. WALDMAN, E. A. Tendência temporal e desigualdades na mortalidade por diarreias em menores de 5 anos. Jornal de Pediatria, 2009. DOI. 0021-7557/08/85-01/21 DOI: 10.1590/S0021-75572009000100005

LANZIERI TM, COSTA I., SHAFI F. A., CUNHA M. H., ORTEGA-BARRIA E., LINHARES A. C., Trends in Hospitalizations From All-Cause Gastroenteritis in Children Younger Than 5 Years of Age in Brazil Before and After Human Rotavirus Vaccine Introduction1998-2007. Ped Inf Dis J. 2010, 673-5.

MORETTIN, P. A. Ondas e Ondaletas: da análise de Fourier à análise de ondaletas. EDUSP, SP, 1999.

NASON, G. P. Wavelet Methods in Statistics with R. Springer, New York, 2008.

DOI: $10.1007 / 978-0-387-75961-6$

PITZER, V.E., VIBOUD, C., SIMONSEN, L., STEINER, C., PANOZZO, C. A., ALONSO, W. J., MILlER, M.A., GLASS, R. I., GLASSER, J.W., PARASHAR, U.D., GRENFELL, B.T. Demographic Variability, Vaccination, and the Spatiotemporal Dynamics of Rotavirus Epidemics. Arigo 2 Science, vol. 325, no.5938, pp. 290-294, 2009. DOI: $10.1126 /$ science. 1172330

SOUZA, E. M., MASUKURA, M. L. T., UCHIMURA, T. T., MORIWAKA, A. M. Intervention Time Series Analysis: Impact of rotavirus vaccine in rates of hospital admissions by acute diarrhea In: Annual Symp: Innovations in Design, Anal, and Dissemination: Frontiers Biost Met, Kansas C, 2013.

von SACHS, R., NASON, G. P., and KROISANDT, G. Wavelet processes and adaptive estimation of the evolutionary wavelet spectrum. Tech. Rep. 516,

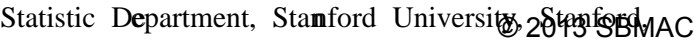
1997. 